\title{
Pure but not simple
}

\author{
Protein purification is stepping into the limelight, as proteomics researchers demand \\ faster ways to purify more proteins. Tim Chapman looks at what is around to help them.
}

$\mathrm{P}$ rotein isolation is one of the oldest 'biotechnologies', but the demands from proteomics for the purification of potentially vast numbers of proteins is driving new developments in long-established techniques. "Researchers working in genomics are looking for the next step to fully understand the results of their sequence analysis - the proteins that the genome expresses - in the context of systems biology," says Anke Cassing, associate director for corporate strategy at Qiagen in Hilden, Germany. "The delicate interplay of proteins is, of course, also of extreme interest to pharmaceutical companies, which are always on the lookout for new drug targets."

There is increasing demand from researchers producing biopharmaceuticals - antibodies and proteins used as drugs. "There's a driving force towards protein purification, separation and analysis," says Carsten Buhlmann, product manager at Agilent, based in Palo Alto, California. "Especially for the biopharmaceuticals, there's a high demand for purity of these proteins that are used for drugs and have to get through all the regulations."

For virtually all applications, researchers need to maintain a protein's biological activity, which can rule out some purification processes. Proteins can be fragile and easily denatured, and many of the most important are insoluble in the most common media.

"If you look at the average protein, it's quite complex, it's a buzzing, vibrating moleculeit's not a fixed structure," says Allan Simpson, vice-president for product development at the protein separations division of GE Healthcare Biosciences in Uppsala, Sweden. "They're very hard to handle, they're difficult to purify, they can aggregate easily — these are very hard things to manipulate."

\section{Proteomics workhorse}

With proteins taking centre stage in many laboratories, equipment developers are rolling out a new generation of automated

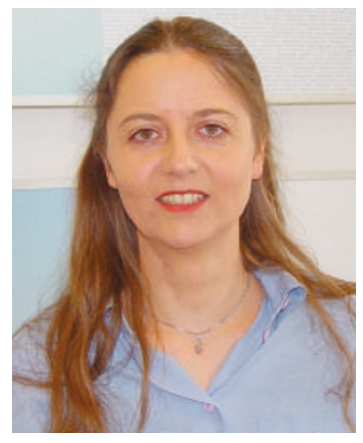

Anke Cassing: systems biology is the next step. systems to take the grind out of separating and purifying proteins of interest. Chromatographic separation is one of the basic proteinpurification techniques and one platform that is emerging as a workhorse of the large proteomics lab is ÄKTAxpress. Made by GE Healthcare, this is a dedicated high-throughput multistep chromatography system for purifying histidine (His)and glutathione-S-transferase (GST)-tagged recombinant proteins.

GE began developing the platform in the late 1990s after realizing that there were not enough trained chromatographers to produce proteins in the quantities and varieties demanded by post-genomic researchers. "We decided to see if we could automate a system that would be better than the current technologies at solving that problem," Simpson says. "Instead of taking a robot and automating the current system, we set out to develop a

\section{SMALL-SCALE SEPARATION}

Sometimes you can do more with less. Proprietary pipette tips developed by PhyNexus in San Jose, California, promise high performance in tiny volumes with minimum fuss. The key lies in encapsulating very small quantities just 5-10 microlitres - of protein separation resin between hydrophilic screens in the very end of the pipette tip. "The whole sample is obliged to make highly intimate contact with that resin," says Chris Hanna, vicepresident of business development. "That results in high trapping efficiency for the sample. We can get a 10-20-fold increase in the target protein

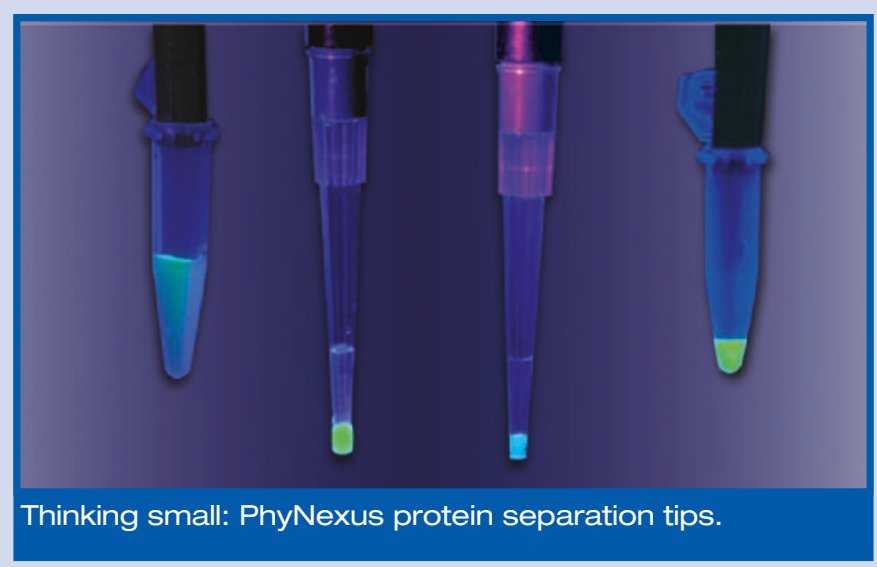

sample from just a few hundred microlitres of sample, and get purities that are often over $95 \%$ with a single separation step."

As well as the technical collaboration with Caliper of Hopkinton, Massachusetts, PhyNexus has designed its PhyTips to be compatible with liquid-handling robots from Tecan of Männedorf, Switzerland, Beckman Coulter of Fullerton, California, and PerkinElmer of Boston, Massachusetts. The firm has also agreed licences to use some of the most advanced resins in its tips, including Qiagen's Ni-NTA resin for purifying histidine-tagged proteins. The combination of tips, resins and platforms makes for exquisite control of the separation process, Hanna says. "You can control the number of cycles that go back and forth through the bed, the rate they do so, the composition of the washes. We can really make that microvolume of material dance and perform at its best," he says. "To be able to do that and maintain fully functional proteins at these very small scales gives you ÄKTA-type purification off a very small amount of starting sample. People can avoid scaling up."

The tips are initially being deployed for rapid purification and enrichment of antibodies from small cultures of Escherichia coli for use in highthroughput cell-based assays, giving significant savings in time and money. "People are wanting to get real biological information much earlier in their screening process, and to do that they have to have the stuff properly prepped," notes Hanna. Interest is also coming from protein engineering and biopharmaceutical companies looking to miniaturize their proteinexpression systems. 
new one. A robot represents nothing more than a mechanical technician - it reproduces all the successes but also all the errors, so you don't move forward in your science."

The company produced a high-throughput system that needs no specialized knowledge to operate and can be programmed to carry out up to four common purification steps starting with affinity purification. "The skill of chromatography is sitting within the system," says Simpson. The software is written as a series of wizards, each representing a single step. GE will shortly be launching a software package for the purification of monoclonal antibodies.

The basic four-module set-up can purify up to 2,500 proteins a year, each module producing up to $50 \mathrm{mg}$ of protein per run. A twin-pack version is aimed at smaller labs wanting to purify up to 1,000 proteins a year.

The new protein-purification facility at Monash University in Melbourne, Australia, is deploying a 12-module ÄKTAxpress setup for its ambitious development programme. "I'm a structural biologist, so my interest is in producing large amounts of recombinant protein for structural and functional studies," says James Whisstock, scientific director of the facility. "What's really important is that the cost of equipment is within reach of a normal university laboratory set-up. There are some very big structural biology institutes with between US\$50 million and \$100 million's worth of industrial-scale protein preparation equipment. From our point of view that's not achievable, but we're bringing in this technology, which is going to make a huge difference to our research."

The ability to deal with many more proteins simultaneously will allow the lab to approach problems differently. "If you have a very challenging protein target and want to try 50 different constructs, at the moment it's really not feasible to do that manually one after the other," Whisstock points out. "Now, you can try the same molecule from 50 different species. With parallel advances in expression technology, the whole process is simplified and really streamlined, and provides the capacity to perform that experiment. You're trying so many different things simultaneously, you're likely to get a result."

\section{Automating innovation}

Several companies are developing equipment and product ranges that can be used at different points in the proteomics pipeline, from raw cell extracts to mass spectrometry and beyond. Beckman Coulter in Fullerton, California, is rolling out its ProteomeLab family to help with everything from initial purification of cell extracts, through protein fractionation and characterization, to the ultimate steps of disease diagnosis.

"We try to link technologies together to simplify the job for what takes place at the end, which is typically mass spectrometry," says John Hobbs, group product manager for ProteomeLab. "To get to that point, a lot of people have realized that it's garbage in, garbage out. If you put crap into a mass spectrometer,

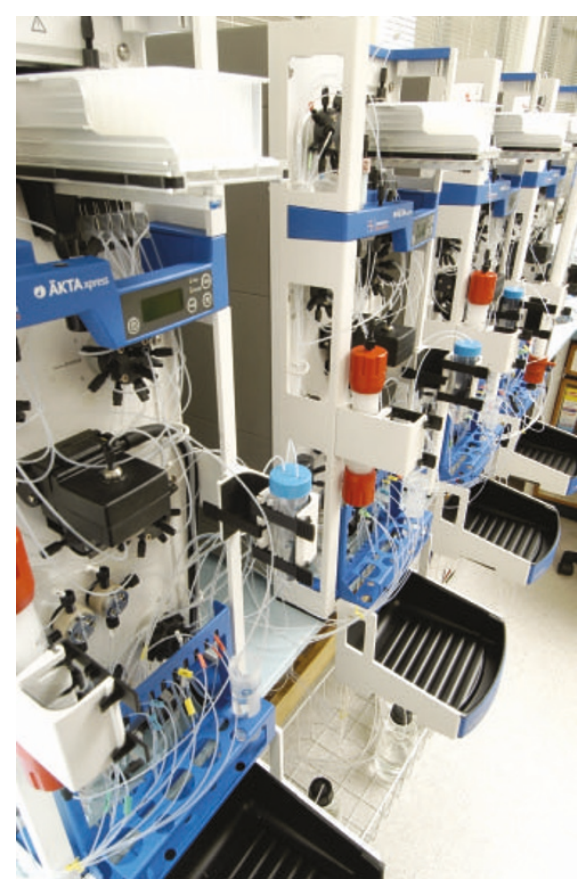

Scaling-up: ÄKTAxpress at Monash University's protein purification facility.

the results you get out will be the same."

The ProteomeLab PF 2D Protein Fractionation System automates two-dimensional chromatographic fractionation, resolving proteins by isoelectric point and hydrophobicity. The emphasis is on standardizing the protocols and techniques used by researchers. "Biologists want to be able to look at their results and see if they relate to someone else's,"

\section{ATTRACTING ATTENTION}

Magnetic beads have been used for protein separation since the 1980s, but the technology is now being adapted for new proteomic applications and use with automated platforms. The market leader in paramagnetic beads is Dynal Biotech based in Oslo, Norway, and recently acquired by life-sciences giant Invitrogen in Carlsbad, California.

Dynal has just signed a co-marketing agreement for its Dynabead kits and Tecan's Freedom EVO automated platform, and has developed protocols for other platforms such as Beckman Coulter's Biomek FX and the KingFisher magnetic separation platform from Thermo Electron of Waltham, Massachusetts. "The main purpose of having magnetic beads is that you can automate the whole process," says Lars Korsnes, director of research and development at Dynal. "The bead technology has some advantages compared with standard chromatography systems - while it's not so easy to put a whole-blood sample into a chromatography column, with magnetic beads you can put the whole sample in."

Dynabeads, like those from some other

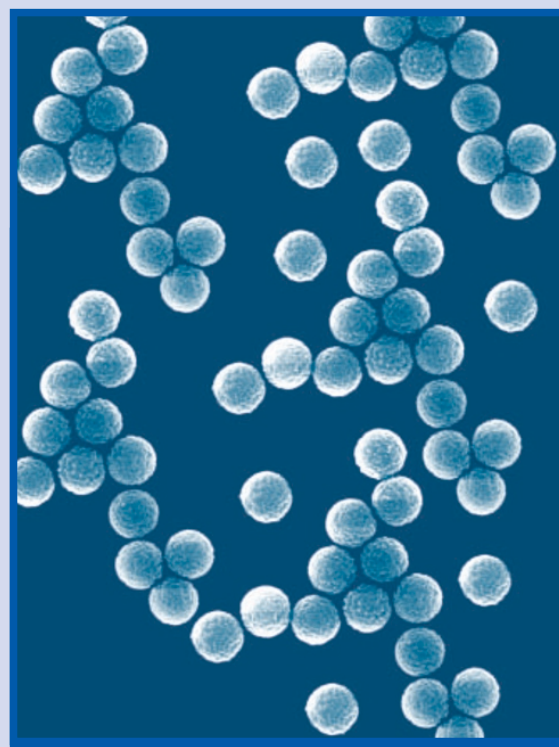

Magnetic beads are branching out. suppliers, are superparamagnetic, with no residual magnetism outside an applied magnetic field. They are also uniform in size, shape and surface properties. This all helps to prevent the beads clogging up an automated device, Korsnes notes.

Bead technology is also more scaleable than chromatography columns, although Dynal is concentrating on more analytical or small-scale protein isolation and protein fractionation for different applications in proteomics. The firm is currently launching a new range of beads with functionalities such as ion-exchange groups, reverse-phase chromatography and hydrophobic chemistries. New owner Invitrogen plans to apply Dynal's surface technologies to a wider range of products.

The beads are also showing promise in the challenging separation of membrane proteins. "There have been some publications where one can bind membrane proteins either before or after lysing the cells," Korsnes says. "Whether we will develop a special protocol is a question for the future, but the technology is there already." 
Hobbs notes. "As well as providing the instrument, we provide the methodology and buffers, but we do specify you have to use that method to get the full support. It's a little bit different from the normal research instrument approach, but we thought there was a need for that and it seems to be accepted."

Beckman is also currently commercializing an innovative system of protein partitioning using affinity fractionation to decrease the unwanted complexity of protein mixtures before analysis. The aim is to remove not just very abundant proteins, for example serum albumin from blood plasma, but also other proteins that are already well characterized. The firm claims that up to $95 \%$ of the proteins in a cell lysate can be removed before the full fractionation stage with less risk than other purification procedures of losing the proteins you're interested in. "There's a growing interest in what might be going away with these large-abundance proteins — albumin is a binding protein, and possibly some interesting proteins go with it," Hobbs notes.

Several big equipment producers have teamed up with smaller specialist firms to include cutting-edge reagents or media in application kits for their automated systems. Tecan in Männedorf, Switzerland, recently signed a licensing agreement to deploy the paramagnetic beads developed by Dynal Biotech in Oslo, Norway, on its Freedom EVO liquid-handling platform (see 'Attracting attention', page 796). The Robopop protein purification kits from Novagen in Madison, Wisconsin, can also be used on
Tecan's workstation and on the MultiPROBE liquid-handling workstation from PerkinElmer in Boston, Massachusetts.

Caliper Life Sciences in Hopkinton, Massachusetts, has integrated into its Sciclone ALH3000 liquid-handling workstation a new column technology developed by PhyNexus based in San Jose, California (see 'Smallscale separation', page 795). The combination allows researchers to purify and enrich small quantities of up to 96 engineered proteins in as little as 15 minutes.

The market for protein purification systems has changed in the past six months, notes Mark Roskey, vice-president of marketing at Caliper, with more groups getting involved in larger-scale protein purification. "It's not at the industrial scale, but regular pharma and biotech R\&D people are now trying to purify proteins in a more parallel situation. A lot of this stems from having all the genes and working with huge numbers of them to develop new drugs," he says.

\section{Analysis and optimization}

To maintain the benefits of high-throughput separation and purification, the proteins of interest must be able to pass smoothly into the next stage of the process. "Once you've got a relatively pure protein you need to determine whether it is pure, so

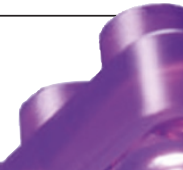

$=\lim _{m}$

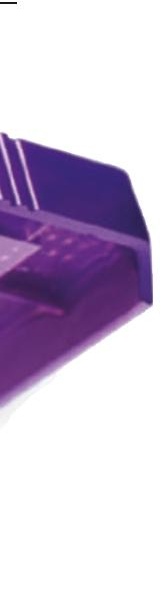

The LabChip90 from Caliper

Life Sciences.

there's issues with analysis as well," says Roskey. A common analysis method is SDSpolyacrylamide gel electrophoresis "but we feel that that is a bottleneck", Roskey adds.

Although the established protocols of macroscale gel electrophoresis are being successfully automated (see 'Automation in two dimensions', below), many users are turning instead to microfluidic and lab-on-a-chip solutions. In January, Caliper launched the Protein Express Assay for its LabChip 90 automated electrophoresis system.

"It's a microfluidic replacement for SDS-PAGE that automates the whole process," Roskey says. "Rather than putting samples on a gel, you get them off a multiwell plate, and it does integrated separation,

\section{AUTOMATION IN TWO DIMENSIONS}

Microfluidic systems have taken over from two-dimensional (2D) gel electrophoresis techniques in some areas of protein separation and analysis, but the established methods are far from dead. Even though they can be slower and messier, the tried and tested 2D protocols still offer some advantages, especially if automation can take out most of the hassle.

"Some people will be claiming otherwise, but I think 2D still has the best levels of sensitivity when you're looking at complex samples," says Paul Orange, senior product manager at NextGen Sciences in Cambridge, UK. "With 2D you're seeing resolution of 2,000-3,000 spots on a gel that's quite a lot of information, but people understand it. Also 2D is a very accessible technology - you can go and buy equipment relatively cheaply and get started if you're looking for a proteomics approach."

In 2003, NextGen launched the first fully automated 2D electrophoresis platform, called a2DE. The firm has now brought out a spin-off system called the a2DEoptimizer which can improve 2D separations on a variety of commercial platforms. "We've not tried to reinvent the wheel," says Orange. "We know what people are using, and know that they have lots of data and experience, but we can help them out by automating key aspects of the process."

The heart of the a2DEoptimizer is automated gel casting, allowing researchers to create customized gradient gel profiles with a minimum of fuss. "Everyone knows casting a gradient gel gives you far superior spot resolution, separation and definition, but the problem is that these gradients can be quite tricky to pour, especially the more exotic ones," Orange says. "There's a very small number of people who can get good reproducible gels when they're pouring gradients. As we see 2D going forward, people are dealing with very small amounts of sample. They've got one or two gels they can run so they have to get the best data they can out of there."

The system also has integrated power packs that can focus samples at high voltages and reduce the time for a separation run. And it has real-time monitoring of the electrical profile. "That's of particular importance, because when you're dealing with new samples, it's very important to look at the electrical profile and tell whether you've got some kind of issue with salt content or protein content," Orange notes. "What we're doing is enabling people to get better data out, and also analyse what's going on in their system." T.C. 
